\section{Treatment of cerebral abscesses}

Despite advances in neurosurgery and chemotherapy, cerebral abscesses still have a disappointingly high mortality rateoften around $40 \%$-and there are even suggestions that it is rising. ${ }^{1-3}$ The inherent difficulties of diagnosing and localising abscesses partly explain the high mortality; but present hopes of improvement come mainly from advances in bacteriology and chemotherapy, as is shown by three papers in this issue (pp 981-987 and 991).

On page 981, de Louvois, Gortavi, and Hurley describe a study by six neurosurgical centres supported by the bacteriology department of Queen Charlotte's Hospital. It required a multicentre study to gather clinical findings from 46 patients with abscesses of the central nervous system, 35 having cerebral abscesses, and to ensure that the bacteriological examinations were carried out by methods which took full account of the difficulties presented by the material-an important detail that has not always been well understood in earlier studies. Thus great emphasis was laid on getting pus from the abscesses into medium of low oxygen tension, and most specimens reached the laboratory within five hours (16 hours was the longest). This is important because anaerobic bacteria with fairly high sensitivity to oxygen are common in cerebral abscesses; in earlier studies they were often not grown because the right conditions were not used. Strict methods of anaerobic culture were ensured in this study, and selective media containing crystal violet, nalidixic acid, or neomycin were inoculated as well as horse-blood agar. Anaerobic streptococci, staphylococci, bacteroides, and enterobacteria were grown in the cultures, and the myth of "sterile" brain abscesses was thus effectively destroyed. Mixed growths were found in seven of 12 temporal lobe abscesses that arose from ear infections. These made up the largest single source of intracranial infection. Bacteroides organisms were isolated from 11 of the 46 abscesses, a figure that might well have been higher if the pus could have reached the examining laboratory even more quickly than it did and if selective medium with nalidixic acid had invariably been used.

The study by Ingham, Selkon, and Roxby ( $p$ 991) paid particular attention to these two matters. Pus went straight from the patient into culture in 30-60 minutes (personal communication from authors) and was invariably grown anaerobically on horse-blood agar containing nalidixic acid at a final concentration of $50 \mu \mathrm{g} \mathrm{ml}$. Aerobic bacteria were isolated from six out of 10 patients and anaerobic bacteria from all of them; at least one species of Bacteroides was consistently present, Bacteroides fragilis in nine cases.

Chemotherapy followed different lines in the two studies. In the multicentre study ( $p$ 981) a variety of antibiotics was used, but not metronidazole. Twenty-three of the 32 patients received penicillin, and the drug was detectable in the pus or serum, or both, from 17 of these patients. Thirteen of 16 patients treated with other $\beta$-lactam antibiotics showed drug in pus or serum, or both; but one patient receiving ampicillin and two receiving cloxacillin gave negative assay results in both pus and serum. During the multicentre study ( $p$ 981) 11 of the 46 patients died (three females and eight males), nine of them after neurosurgery - a mortality rate of $20 \%$ in treated cases. Among the 35 patients with brain abscesses seven died, again a mortality rate of $20 \%$, but only five $(14 \%)$ in the treated cases. These low figures are encouraging.
Ingham et al ( $\mathrm{p}$ 991) had 10 patients, one of whom was dead on arrival at hospital. The other nine received metronidazole (400-600 mg 8-hourly) by mouth or intravenously, penicillin ( $0.6 \mathrm{~g} \mathrm{6}$-hourly) or ampicillin ( $0.5 \mathrm{~g} 6$-hourly), and gentamicin ( $80 \mathrm{mg} 8$-hourly), the regimen being later modified according to the bacterial flora of the abscess. All their 10 patients had otogenic cerebral abscesses, so their results are not comparable with those of the multicentre study. They are, however, striking results: eight of the nine treated patients made remarkably rapid and full recoveries, and the other survived with residual cerebellar ataxia after a complicated postoperative course. In four patients metronidazole was found in pus or ventricular fluid in concentrations ranging between 20.7 and $45.0 \mu \mathrm{g} \mathrm{ml}$. Since the minimal bactericidal concentration of metronidazole for Bacteroides fragilis is $6.25 \mu \mathrm{g} \mathrm{ml}$ these are highly satisfactory concentrations at the seat of infection.

If the apparent predominance of Bacteroides fragilis and the efficacy of metronidazole treatment are confirmed, the prospects may indeed be regarded as hopeful. An ad hoc investigation on the lines of the multicentre study would be the best way to confirm these results.

${ }^{1}$ Garfield, J, British Medical fournal, 1969, 2, 7.
2 British Medical fournal, 1969, 2, 1.

${ }^{3}$ British Medical fournal, 1975, 3, 504.

\section{Current thoughts on surgery for portal hypertension}

The disappointing outcome of recent controlled trials ${ }^{1-3}$ of portacaval anastomosis (PCA) may suggest to clinicians that nothing can be done to improve survival in patients with portal hypertension. But have we enough information from which to draw such conclusions? Certainly there seems to be no justification for prophylactic portacaval shunts in patients who have never bled from their varices. Three studies have shown conclusively that survival is no different in the operated group and one showed it to be worse-the decreased frequency of bleeding is offset by the increased frequency of hepatic encephalopathy and liver failure. ${ }^{1-3}$

The value of PCA in those patients who have recently bled or are continuing to bleed (a somewhat arbitrary distinction) has also been the subject of extensive research. Orloff ${ }^{4}$ continues to advocate emergency shunting in spite of a $52 \%$ operative mortality rate, but most surgeons prefer to operate electively some time after the patient has stopped bleeding. Two randomised controlled trials of such patients, one from the Veterans' Administration co-operative study ${ }^{5}$ and the other from the Boston Inter-Hospital Liver Group, ${ }^{6}$ have shown five-year survival rates of $55 \%$ and $56 \%$ respectively for the surgical group and $34 \%$ and $48 \%$ for the non-operated group, differences that were not statistically significant. In a similar trial from France Rueff $^{7}$ reported a higher mortality in the shunted group. The relative failure of PCA to increase the survival rate was once again associated with a higher incidence of disabling encephalopathy in the surgical group $(20 \%$ after one year and $50 \%$ after five years).

There are, however, many inherent difficulties present in setting up such trials that need to be considered before far-reaching conclusions are drawn. Most of these 